\title{
Entanglement-Assisted Local Manipulation of Pure Quantum States
}

\author{
Daniel Jonathan and Martin B. Plenio \\ Blackett Laboratory, Imperial College, London SW7 2BZ, United Kingdom
}

(Received 24 May 1999)

\begin{abstract}
We demonstrate that local transformations on a composite quantum system can be enhanced in the presence of certain entangled states. These extra states act much like catalysts in a chemical reaction: they allow otherwise impossible local transformations to be realized, without being consumed in any way. In particular, we show that this effect can considerably improve the efficiency of entanglement concentration procedures for finite states.

PACS numbers: 03.67.Hk, 89.70.+c
\end{abstract}

The rapid development of quantum information processing in recent years has led us to view quantummechanical entanglement as a useful physical resource [1]. As with any such resource, there arises naturally the question of how it can be quantified and manipulated. Attempts have been made to find meaningful measures of entanglement [2-6], and also to uncover the fundamental laws of its behavior under local quantum operations and classical communication (LQCC) [2-12]. These laws are fundamentally and also practically important, since many applications of quantum information processing involve spatially separated parties who must manipulate an entangled state without performing joint operations. In this context, it is generally assumed that entanglement may be used to perform useful tasks only if it is consumed in whole or in part. Indeed, this is implicit in the commonsense notion of a "resource."

In this Letter we demonstrate that entanglement is, in fact, a stranger kind of resource, one that can be used without being consumed at all. More precisely, we show that the mere presence of an entangled state can allow distant parties to realize local transformations that would otherwise be impossible, or less efficient. Our idea is best introduced by the following situation, illustrated in Fig. 1. Imagine that Alice and Bob share a finitedimensional entangled state $\left|\psi_{1}\right\rangle$ of two particles, which they would like to convert, using only LQCC, into the state $\left|\psi_{2}\right\rangle$. For some choices of $\left|\psi_{1}\right\rangle$ and $\left|\psi_{2}\right\rangle$ there exists a local protocol that accomplishes this task with certainty [9], but for others it can be done only probabilistically, with some maximum probability $p_{\max }<1$ [10]. Assume the latter is the case, as indicated by the crossed arrow in the upper part of Fig. 1. Now suppose that an "entanglement banker," let us call him Scrooge, agrees to lend Alice and Bob another entangled pair of particles $|\phi\rangle$, under the condition that exactly the same state must be returned to him later on. Given this additional state, will Alice and Bob be able to transform $\left|\psi_{1}\right\rangle$ into $\left|\psi_{2}\right\rangle$ and still return the state $|\phi\rangle$ to Scrooge? We suggest to call a transformation of this kind, which uses intermediate entanglement without consuming it, an entanglement-assisted local transformation, abbreviated by ELQCC. The possible existence of such a class of transformations has been conjectured by Popescu [13] (see also [14]).

The main result of this Letter is the proof that entanglement-assisted local transformations are indeed more powerful than ordinary local transformations. This result is significant in a number of ways. First of all, it provides a concrete mechanism by which Alice and Bob can enhance their entanglement-manipulation ability. For example, we will demonstrate that entanglement concentration is more efficient with ELQCC than with only LQCC. Moreover, the definition of a meaningful new class of entanglement transformations demonstrates that the structure of entanglement, even for pure, bipartite states, is still not completely understood.

Let us begin then with an explicit example of the power of entanglement-assisted transformations. The central tool we will require for this is Nielsen's theorem $[9,11]$.

Theorem (Nielsen): Let $\left|\psi_{1}\right\rangle=\sum_{i=1}^{n} \sqrt{\alpha_{i}}\left|i_{A}\right\rangle\left|i_{B}\right\rangle$ and $\left|\psi_{2}\right\rangle=\sum_{i=1}^{m} \sqrt{\alpha_{i}^{\prime}}\left|i_{A}\right\rangle\left|i_{B}\right\rangle$ be pure bipartite states, with Schmidt coefficients [15], respectively, $\alpha_{1} \geq \ldots \geq$ $\alpha_{n}>0$ and $\alpha_{1}^{\prime} \geq \ldots \geq \alpha_{m}^{\prime}>0$ (we can refer to such
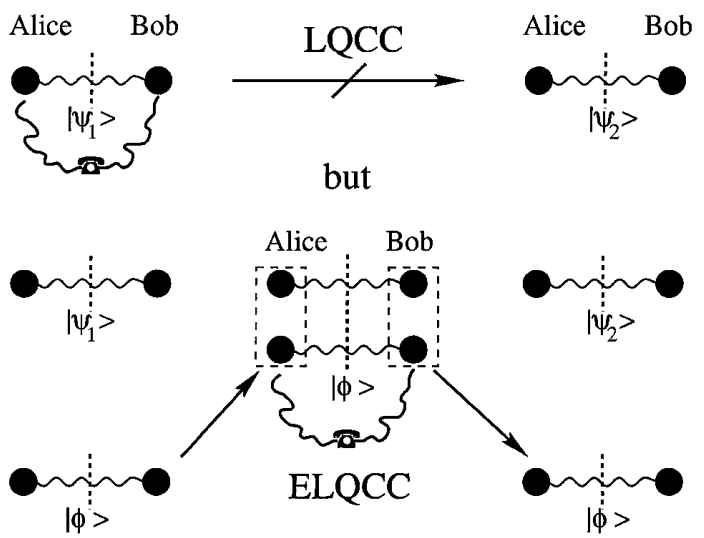

FIG. 1. Alice and Bob share a finite bipartite system in state $\left|\psi_{1}\right\rangle$. Using only LQCC they are not able to convert this state into $\left|\psi_{2}\right\rangle$ with certainty. However, if they are temporarily supplied with another entangled state $|\phi\rangle$, they can always achieve the transformation from $\left|\psi_{1}\right\rangle$ to $\left|\psi_{2}\right\rangle$. The state $|\phi\rangle$ is not consumed and can therefore be viewed as a catalyst for this transformation. 
distributions as "ordered Schmidt coefficients," or OSCs). Then a transformation $T$ that converts $\left|\psi_{1}\right\rangle$ to $\left|\psi_{2}\right\rangle$ with $100 \%$ probability can be realized using LQCC iff the OSCs $\left\{\alpha_{i}\right\}$ are majorized [16] by $\left\{\alpha_{i}^{\prime}\right\}$, that is, iff for $1 \leq l \leq n$

$$
\sum_{i=1}^{l} \alpha_{i} \leq \sum_{i=1}^{l} \alpha_{i}^{\prime}
$$

One consequence of Nielsen's theorem is that there exist pairs $\left|\psi_{1}\right\rangle$ and $\left|\psi_{2}\right\rangle$ where neither state is convertible into the other with certainty under LQCC. Such pairs are called incomparable [9], and can be indicated by $\left|\psi_{1}\right\rangle \leftrightarrow\left|\psi_{2}\right\rangle$. Examples are the following two states:

$$
\begin{aligned}
& \left|\psi_{1}\right\rangle=\sqrt{0.4}|00\rangle+\sqrt{0.4}|11\rangle+\sqrt{0.1}|22\rangle+\sqrt{0.1}|33\rangle, \\
& \left|\psi_{2}\right\rangle=\sqrt{0.5}|00\rangle+\sqrt{0.25}|11\rangle+\sqrt{0.25}|22\rangle .
\end{aligned}
$$

It can easily be checked that $\alpha_{1}<\alpha_{1}^{\prime}$ but $\alpha_{1}+\alpha_{2}>$ $\alpha_{1}^{\prime}+\alpha_{2}^{\prime}$, so indeed $\left|\psi_{1}\right\rangle \nLeftarrow\left|\psi_{2}\right\rangle$. If Alice and Bob share one of these states and wish to convert it to the other using LQCC, they must therefore run the risk of failure. Their greatest probability of success is given by [10]

$$
p_{\max }\left(\left|\psi_{1}\right\rangle \rightarrow\left|\psi_{2}\right\rangle\right)=\left.\min \right|_{1 \leq l \leq n} \frac{E_{l}\left(\left|\psi_{1}\right\rangle\right)}{E_{l}\left(\left|\psi_{2}\right\rangle\right)},
$$

where $E_{l}\left(\left|\psi_{1}\right\rangle\right)=1-\sum_{i=1}^{l-1} \alpha_{i}$. For instance, in the case of the pair in Eq. (2), $p_{\max }$ is only $80 \%$.

Suppose now that Scrooge lends them the 2-qubit state

$$
|\phi\rangle=\sqrt{0.6}|44\rangle+\sqrt{0.4}|55\rangle .
$$

The Schmidt coefficients $\gamma_{k}, \gamma_{k}^{\prime}$ of the product states $\left|\psi_{1}\right\rangle|\phi\rangle,\left|\psi_{2}\right\rangle|\phi\rangle$, given in decreasing order, are

$$
\left|\psi_{1}\right\rangle|\phi\rangle: 0.24,0.24,0.16,0.16,0.06,0.06,0.04,0.04,
$$

$$
\left|\psi_{2}\right\rangle|\phi\rangle: 0.30,0.20,0.15,0.15,0.10,0.10,0.00,0.00 \text {. }
$$

so that $\sum_{i=1}^{k} \gamma_{k} \leq \sum_{i=1}^{k} \gamma_{k}^{\prime}, 1 \leq k \leq 8$. Nielsen's theorem then implies that the transformation $\left|\psi_{1}\right\rangle|\phi\rangle \rightarrow$ $\left|\psi_{2}\right\rangle|\phi\rangle$ can, in fact, be realized with $100 \%$ certainty using LQCC. Alice and Bob can complete their task and still return the borrowed state $|\phi\rangle$ to Scrooge. This state acts therefore much like a catalyst in a chemical reaction: its presence allows a previously forbidden transformation to be realized, and since it is not consumed it can be reused as many times as desired. This represents a fundamental distinction between the present effect and previous proposals for using entanglement as an enhancing factor, such as entanglement pumping [17] or the activation of bound entanglement [18], where the extra entanglement was either used up or transformed. We shall thus adopt the "catalysis" metaphor as a convenient way of referring to our novel effect.

Nielsen's theorem, along with its generalization given in [11], provides a complete answer to the question "which transformations on a pure bipartite state are possible un- der LQCC?" It would, of course, be desirable to find analogous conditions for ELQCC. For instance, given $\left|\psi_{1}\right\rangle,\left|\psi_{2}\right\rangle$, we would like to know when there exists an appropriate catalyst state $|\phi\rangle$. Mathematically, this means that given the OSCs $\left\{\alpha_{i}\right\},\left\{\alpha_{i}^{\prime}\right\}$, we have to determine when there exists other OSCs $\left\{\beta_{k}\right\}$, such that $\left\{\alpha_{i} \beta_{k}\right\}$ is majorized by $\left\{\alpha_{j}^{\prime} \beta_{k}\right\}$. Unfortunately, this problem seems in general to be hard to solve analytically [19]. The difficulty lies in the fact that, before Nielsen's theorem can be applied to the tensor products, their Schmidt coefficients must be sorted into descending order. No general analytic way for doing this is known, so we are at present confined to searching numerically for appropriate catalysts. Nevertheless, it is possible to present a few interesting partial results.

Lemma 1: No transformation can be catalyzed by a maximally entangled state $\left|\varphi_{p}\right\rangle=(1 / \sqrt{p}) \sum_{i=1}^{p}\left|i_{A}\right\rangle\left|i_{B}\right\rangle$.

Proof: The Schmidt coefficients $\gamma_{j}$ of $\left|\psi_{1}\right\rangle\left|\varphi_{p}\right\rangle$ are just $\frac{\alpha_{i}}{p}$, each one being $p$-fold degenerate. In this case sorting them is trivial, and we can write that, for any $l, \sum_{j=1}^{p l} \gamma_{j}=\sum_{i=1}^{l} \alpha_{i}$. Now, by Nielsen's theorem, if $\left|\psi_{1}\right\rangle \nrightarrow\left|\psi_{2}\right\rangle$ under LQCC, then for some $l=l_{0}$ we have $\quad \sum_{i=1}^{l_{0}} \alpha_{i}>\sum_{i=1}^{l_{0}} \alpha_{i}^{\prime} \Rightarrow \sum_{j=1}^{p l_{0}} \gamma_{j}>\sum_{j=1}^{p l_{0}} \gamma_{j}^{\prime} \Rightarrow$ $\left|\psi_{1}\right\rangle\left|\varphi_{p}\right\rangle \nrightarrow\left|\psi_{2}\right\rangle\left|\varphi_{p}\right\rangle$ under LQCC.

This result shows a surprising property of catalysts: they must be partially entangled. Roughly speaking, if the catalyst has "not enough" entanglement, Alice and Bob will not be able to transform $\left|\psi_{1}\right\rangle$ into $\left|\psi_{2}\right\rangle$ with certainty, but if it has "too much" then they will not be able to return it intact to Scrooge.

Lemma 2: Two states are interconvertible (i.e., both $\left|\psi_{1}\right\rangle \rightarrow\left|\psi_{2}\right\rangle$ and $\left.\left|\psi_{2}\right\rangle \rightarrow\left|\psi_{1}\right\rangle\right)$ under ELQCC iff they are equivalent up to local unitary transformations.

Proof: Suppose that $\left|\psi_{1}\right\rangle \leftrightarrow\left|\psi_{2}\right\rangle$ under ELQCC. Then there exist $|\eta\rangle,|\phi\rangle$ such that both $\left|\psi_{1}\right\rangle|\phi\rangle \rightarrow\left|\psi_{2}\right\rangle|\phi\rangle$ and $\left|\psi_{2}\right\rangle|\eta\rangle \rightarrow\left|\psi_{1}\right\rangle|\eta\rangle$ are possible under LQCC. This means that $\left|\psi_{1}\right\rangle|\phi\rangle|\eta\rangle$ and $\left|\psi_{2}\right\rangle|\phi\rangle|\eta\rangle$ are interconvertible under LQCC, which happens iff their Schmidt coefficients are identical $[8,9]$. This in turn implies that the Schmidt coefficients of $\left|\psi_{1}\right\rangle$ and $\left|\psi_{2}\right\rangle$ are also identical, and thus that they are equivalent under local unitary rotations.

One consequence of Lemma 2 is that if a transition that is forbidden under LQCC can be catalyzed by extra entanglement (i.e., $\left|\psi_{1}\right\rangle \nrightarrow\left|\psi_{2}\right\rangle$ under LQCC but $\left|\psi_{1}\right\rangle \rightarrow\left|\psi_{2}\right\rangle$ under ELQCC), then the reverse transition (from $\left|\psi_{2}\right\rangle$ to $\left.\left|\psi_{1}\right\rangle\right)$ must be impossible even under ELQCC. In particular, only transitions between incomparable states may be catalyzed. Therefore, catalysis is impossible if $\left|\psi_{1}\right\rangle$ and $\left|\psi_{2}\right\rangle$ are both $2 \times 2$ states, for in this case it is always true that either $\left|\psi_{1}\right\rangle \rightarrow\left|\psi_{2}\right\rangle$ or $\left.\left|\psi_{2}\right\rangle \rightarrow \psi_{1}\right\rangle$ under LQCC.

A somewhat more surprising result is that catalysis is also impossible when $\left|\psi_{1}\right\rangle$ and $\left|\psi_{2}\right\rangle$ are both $3 \times 3$ states. In this case incomparable states do exist [9], so Lemma 2 does not immediately rule it out. To see that it actually cannot occur, we must look more closely at the relevant Schmidt coefficients.

Lemma 3: Let $\left|\psi_{1}\right\rangle,\left|\psi_{2}\right\rangle$ be $n \times n$-level states, with OSCs $\left\{\alpha_{i}\right\},\left\{\alpha_{i}^{\prime}\right\}, 1 \leq i \leq n$. Then $\left|\psi_{1}\right\rangle \rightarrow\left|\psi_{2}\right\rangle$ under 
ELQCC only if both

$$
\alpha_{1} \leq \alpha_{1}^{\prime}, \quad \alpha_{n} \geq \alpha_{n}^{\prime} .
$$

Proof: Let $\{\beta\}_{j=1}^{m}$ be the OSCs of $|\phi\rangle$. Then the largest and smallest Schmidt coefficients of $\left|\psi_{1}\right\rangle|\phi\rangle$ are, respectively, $\gamma_{1}=\alpha_{1} \beta_{1}$ and $\gamma_{n m}=\alpha_{n} \beta_{m}$ (analogous expressions hold for $\left.\left|\psi_{2}\right\rangle|\phi\rangle\right)$. Nielsen's theorem now tells us that if $\left|\psi_{1}\right\rangle|\phi\rangle \rightarrow\left|\psi_{2}\right\rangle|\phi\rangle$ under LQCC, then $\gamma_{1} \leq$ $\gamma_{1}^{\prime}$ and $\sum_{k=1}^{n m-1} \gamma_{k}=1-\gamma_{n m} \leq \sum_{k=1}^{n m-1} \gamma_{k}^{\prime}=1-\gamma_{n m}^{\prime}$, from which Eq. (6) follows.

Suppose now that $\left|\psi_{1}\right\rangle$ and $\left|\psi_{2}\right\rangle$ are incomparable $3 \times 3$ states. Then Nielsen's theorem implies that one of two possibilities must hold: either

$$
\left\{\begin{array} { l } 
{ \alpha _ { 1 } > \alpha _ { 1 } ^ { \prime } } \\
{ \alpha _ { 1 } + \alpha _ { 2 } < \alpha _ { 1 } ^ { \prime } + \alpha _ { 2 } ^ { \prime } }
\end{array} \text { or } \left\{\begin{array}{l}
\alpha_{1}<\alpha_{1}^{\prime} \\
\alpha_{1}+\alpha_{2}>\alpha_{1}^{\prime}+\alpha_{2}^{\prime}
\end{array}\right.\right.
$$

In either case, Eq. (6) is violated, so $\left|\psi_{1}\right\rangle \nLeftarrow\left|\psi_{2}\right\rangle$ under ELQCC. In other words, there are pairs of states which are incomparable even in the presence of extra entanglement.

In the $4 \times 4$ case, we have seen by example [Eq. (2)] that catalysis is indeed possible. Lemma 3 shows that the only case where it can happen is when the following conditions are all satisfied:

$$
\alpha_{1} \leq \alpha_{1}^{\prime}, \quad \alpha_{1}+\alpha_{2}>\alpha_{1}^{\prime}+\alpha_{2}^{\prime}, \quad \alpha_{4} \geq \alpha_{4}^{\prime},
$$

where the second condition ensures that the transformation is not possible under LQCC alone. Indeed, the states $\left|\psi_{1}\right\rangle,\left|\psi_{2}\right\rangle$ in Eq. (2) are of this type.

The concept of entanglement-assisted transformations may be extended in a number of ways. An example is when the presence of a catalyst state does not allow Alice and Bob to transform $\left|\psi_{1}\right\rangle$ into $\left|\psi_{2}\right\rangle$ with certainty, but still increases the optimal probability with which this can be done. For instance, consider the incomparable $3 \times$ 3 states $\left|\psi_{1}\right\rangle=\sqrt{0.5}|00\rangle+\sqrt{0.4}|11\rangle+\sqrt{0.1}|22\rangle$ and $\left|\psi_{2}\right\rangle=\sqrt{0.6}|00\rangle+\sqrt{0.2}|11\rangle+\sqrt{0.2}|22\rangle$. From Eq. (3) the optimal probability of converting $\left|\psi_{1}\right\rangle$ into $\left|\psi_{2}\right\rangle$ under LQCC is $80 \%$, and Lemma 3 tells us that this cannot be increased to $100 \%$ by the use of any catalyst. Nevertheless, Eq. (3) also shows that $p_{\max }\left(\left|\psi_{1}\right\rangle|\phi\rangle \rightarrow\left|\psi_{2}\right\rangle|\phi\rangle\right)$ can be as large as $90.04 \%$ when $|\phi\rangle=\sqrt{0.65}|33\rangle+\sqrt{0.35} 44\rangle$.

Even this limited enhancement is not always possible, as shown by the following result.

Lemma 4: Let $\left|\psi_{1}\right\rangle,\left|\psi_{2}\right\rangle$ be $n \times n$ bipartite states with OSCs $\left\{\alpha_{i}\right\},\left\{\alpha_{i}^{\prime}\right\}$, and such that $p_{\max }\left(\left|\psi_{1}\right\rangle \rightarrow\left|\psi_{2}\right\rangle\right)$ under LQCC is $\frac{\alpha_{n}}{\alpha_{n}^{\prime}}$. Then this probability cannot be increased by the presence of any catalyst state.

Proof: Let $|\phi\rangle$ be a bipartite state with OSCs $\{\beta\}_{i=1}^{m}$. From Eq. (3), the optimal probability of converting $\left|\psi_{1}\right\rangle|\phi\rangle$ into $\left|\psi_{2}\right\rangle|\phi\rangle$ under LQCC is given by

$$
p_{\max }=\min _{l \leq n m} \frac{E_{l}\left(\left|\psi_{1}\right\rangle|\phi\rangle\right)}{E_{l}\left(\left|\psi_{2}\right\rangle|\phi\rangle\right)} \leq \frac{E_{n m}\left(\left|\psi_{1}\right\rangle|\phi\rangle\right)}{E_{n m}\left(\left|\psi_{2}\right\rangle|\phi\rangle\right)}=\frac{\alpha_{n}}{\alpha_{n}^{\prime}},
$$

where we have used that $E_{n m}\left(\left|\psi_{1}\right\rangle|\phi\rangle\right)=\alpha_{n} \beta_{m}$.

In particular, Lemma 4 applies when $\left|\psi_{1}\right\rangle$ has $n$ Schmidt coefficients and $\left|\psi_{2}\right\rangle$ is the maximally entangled state $\left|\varphi_{n}\right\rangle$, for in this case $p_{\max }\left(\left|\psi_{1}\right\rangle \rightarrow\left|\varphi_{n}\right\rangle\right)=n \alpha_{n}[7,10]$. At first sight, this may seem to indicate that catalytic effects cannot increase the efficiency with which entanglement can be concentrated into maximally entangled form. It turns out, however, that the opposite is actually the case. To see this, recall first that an entanglement concentration protocol (ECP) can be defined $[3,11]$ as any sequence of LQCC's that transform a given partially entangled state $\left|\psi_{1}\right\rangle$ into a maximally entangled state $\left|\varphi_{m}\right\rangle$ of $m$ levels, with probability $p_{m}$ (note that $\left|\varphi_{1}\right\rangle$ is a disentangled state). Among all these protocols, the optimal is the one that yields on average the greatest amount of concentrated entanglement, i.e., that maximizes $\langle E\rangle=\sum_{m=1}^{n} p_{m} \ln m$ over all possible distributions $\left\{p_{m}\right\}$ compatible with LQCC. The maximum value $\langle E\rangle_{\max }$ is the (finite) distillable entanglement of $\left|\psi_{1}\right\rangle$ [20]. In [11,12] it was shown that $\langle E\rangle_{\max }=$ $\sum_{m=0}^{n} m \ln m\left(\alpha_{m}-\alpha_{m+1}\right)$, corresponding to a probability distribution $p_{m}^{\mathrm{opt}}=m\left(\alpha_{m}-\alpha_{m+1}\right)$, where $\left\{\alpha_{i}\right\}$ are the OSCs of $\left|\psi_{1}\right\rangle$ and $a_{n+1}=0$.

A catalyzed ECP (Fig. 2) is then any sequence of LQCCs that transform the product $\left|\psi_{1}\right\rangle|\phi\rangle$ (for some catalyst state $|\phi\rangle)$ into one of the states $\left|\varphi_{m}\right\rangle|\phi\rangle$, with probability $p_{m}$. It turns out that in this case the distillable entanglement $\langle E\rangle_{\max }(|\phi\rangle)$ can be larger than the value given above. To show this, we use a general technique for optimizing entanglement transformations, presented in [11]. From the generalized Nielsen's theorem [11], a catalyzed ECP with probability distribution $p_{m}$ can be realized using LQCCs iff the following constraints are satisfied for $1 \leq l \leq n$ :

$$
\sum_{m=1}^{n} p_{m} E_{l}\left(\left|\varphi_{m}\right\rangle|\phi\rangle\right) \leq E_{l}\left(\left|\psi_{1}\right\rangle|\phi\rangle\right)
$$

where $E_{l}$ is the same as in Eq. (3). The optimal protocol can then be found by maximizing $\langle E\rangle(|\phi\rangle)$ with respect

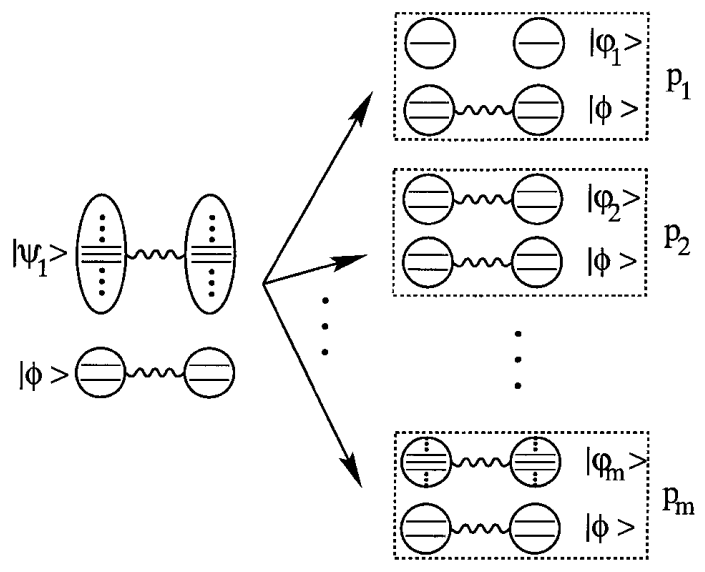

FIG. 2. A catalyzed entanglement concentration protocol. Alice and Bob share a state $\left|\psi_{1}\right\rangle|\phi\rangle$ and by LQCC convert it with probability $p_{m}$ into a product $\left|\varphi_{m}\right\rangle|\phi\rangle$ between a maximally entangled state of $m$ levels and the catalyst state $|\phi\rangle$. The entanglement in $\left|\psi_{1}\right\rangle$ may be concentrated with greater efficiency in this way than in the absence of $|\phi\rangle$. 
to $p_{m}$, given these constraints. This is a standard linear programming problem [21], for which an exact solution can always be found in any particular case. In Fig. 3 we plot $\langle E\rangle_{\max }(|\phi\rangle)$ for the case where $\left|\psi_{1}\right\rangle$ is a $3 \times 3$ state with Schmidt coefficients $\alpha_{1}=0.5, \alpha_{2}=0.3, \alpha_{3}=0.2$, and where $|\phi\rangle$ is a $2 \times 2$ state. We can see that some catalysts allow a substantial increase in the entanglement yield relative to the one achievable using only LQCC.

How does this happen, even under the constraints implied by Lemma 4? It turns out that, although Lemma 4 forbids $p_{n}$ from increasing in the presence of a catalyst, the same is not true for $p_{n-1}$. For instance, in the example above the optimal probability distribution without a catalyst (i.e., with a disentangled catalyst) is [11] $p_{3}^{\text {opt }}=0.6, p_{2}{ }^{\text {opt }}=p_{1}^{\text {opt }}=0.2$. On the other hand, in the presence of a catalyst with Schmidt components $\beta_{1}=$ $0.5825, \beta_{2}=0.4175$, it becomes $p_{3}^{\mathrm{opt}}=0.6, p_{2}^{\mathrm{opt}}=$ $0.3178, p_{1}^{\text {opt }}=0.0822$. Effectively, the presence of a catalyst allows us to syphon probability away from the unwanted outcome where all the entanglement is lost and into one where a maximally entangled state is obtained.

How far can this enhancing effect be used to increase the distillable entanglement $\langle E\rangle_{\max }(|\phi\rangle)$ ? Lemma 4 gives us immediately the following upper bound:

$$
B \equiv n \alpha_{n} \ln n+\left(1-n \alpha_{n}\right) \ln (n-1) \geq\langle E\rangle_{\max }(|\phi\rangle) .
$$

This simply corresponds to a case where $p_{n}$ is maximum, and all the remaining probability is assigned to obtaining $\left|\varphi_{n-1}\right\rangle$. Another upper bound is the asymptotic distillable entanglement per copy [20]. These bounds

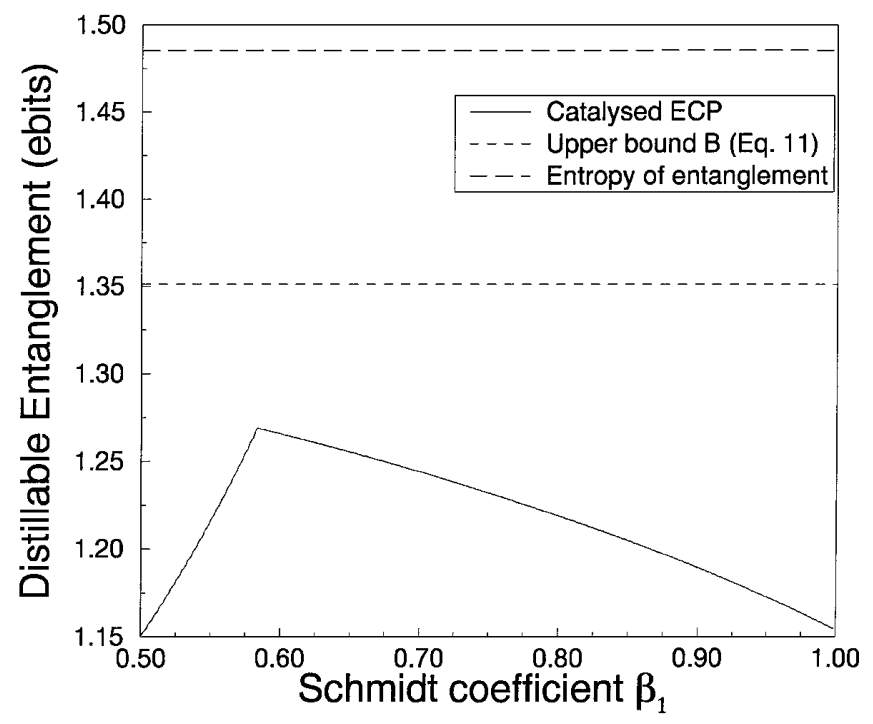

FIG. 3. Finite distillable entanglement $\langle E\rangle_{\max }(|\phi\rangle)$ (in ebits) of the $3 \times 3$ state $\left|\psi_{1}\right\rangle=\sqrt{0.5}|00\rangle+\sqrt{0.3}|11\rangle+\sqrt{0.2}|22\rangle$, in the presence of a catalyst state $|\phi\rangle$ with Schmidt coefficients $\beta_{1} \geq \beta_{2}$. The horizontal lines are upper bounds to $\langle E\rangle_{\max }(|\phi\rangle)$ given by Eq. (12) and by the entropy of entanglement of $\left|\psi_{1}\right\rangle$. Note that neither product states nor maximally entangled states are useful as catalysts. are unrelated: there are states like the one in Fig. 3, for which $B<S$, and others for which $S<B$. It is an open question whether any of these bounds can in general be reached by the use of an appropriate catalyst.

To summarize, we have presented a counterintuitive effect by means of which local entanglement transformations of finite states may be catalyzed in the presence of "borrowed" entanglement. Our results raise many interesting questions. For instance, what are sufficient conditions for the existence of catalysts? Are catalysts always more efficient as their dimension increases? We hope that the intricacy of this effect may convince readers that there is more to pure-state entanglement than just asymptotic properties, and that no single "measure" of entanglement can fully capture it all.

We thank I. Olkin, A. Uhlmann, and O. Pretzel for helpful comments on majorization and tensor products and especially S. Popescu for inspiring discussions. We acknowledge the support of the Brazilian agency Conselho Nacional de Desenvolvimento Científico e Tecnológico (CNPQ), the ORS Award Scheme, the United Kingdom Engineering and Physical Sciences Research Council, the Leverhulme Trust, the European Science Foundation, and the European Union.

[1] M.B. Plenio and V. Vedral, Contemp. Phys. 39, 431 (1998).

[2] C. H. Bennett et al., Phys. Rev. A 54, 3824 (1996).

[3] C.H. Bennett et al., Phys. Rev. A 53, 2046 (1996).

[4] C. H. Bennett et al., Phys. Rev. Lett. 76, 722 (1996).

[5] V. Vedral et al., Phys. Rev. Lett. 78, 2275 (1997).

[6] V. Vedral and M. B. Plenio, Phys. Rev. A 57, 1619 (1998).

[7] H.-K. Lo and S. Popescu, quant-ph/9707038.

[8] G. Vidal, quant-ph/9807077.

[9] M. A. Nielsen, Phys. Rev. Lett. 83, 436 (1999).

[10] G. Vidal, Phys. Rev. Lett. 83, 1046 (1999).

[11] D. Jonathan and M. B. Plenio, Phys. Rev. Lett. 83, 1455 (1999).

[12] L. Hardy, Phys. Rev. A 60, 1912 (1999).

[13] S. Popescu (private communciation).

[14] C. H. Bennett et al., quant-ph/9908073.

[15] Note that the "Schmidt coefficients" defined here are the squares of the usually defined Schmidt coefficients.

[16] A. W. Marshall and I. Olkin, Inequalities: Theory of Majorization and its Applications (Academic Press, New York, 1979).

[17] D. Deutsch et al., Phys. Rev. Lett. 77, 2818 (1996).

[18] P. Horodecki et al., Phys. Rev. Lett. 82, 1056 (1999).

[19] I. Olkin and A. Uhlmann (private communication). Indeed, there does not seem to have been any extensive work by mathematicians on the subject of majorization and tensor products.

[20] $\langle E\rangle_{\max }$ is always less than the asymptotic distillable entanglement, which is the entropy of entanglement $S=$ $-\sum \alpha_{i} \ln \alpha_{i}$ [3]. They become equal in the limit where $\left|\psi_{1}\right\rangle$ consists of $N \rightarrow \infty$ copies of some state $\left|\psi^{\prime}\right\rangle$ [11].

[21] G. R. Walsh, An Introduction to Linear Programming (J. Wiley \& Sons, Chichester, 1983). 